\section{PADRÃO ALIMENTAR DE MULHERES COM CÂNCER DE MAMA: UM ESTUDO $A$ POSTERIORI}

\author{
Dietary pattern of women with breast cancer: a posteriori study \\ Patrón alimentario de mujeres con cáncer de mama: estudio \\ a Posteriori
}

\section{RESUMO}

Objetivo: Identificar o padrão alimentar a posteriori de mulheres com câncer de mama. Métodos: Estudo observacional, transversal e analítico. Foram avaliadas 100 mulheres com câncer de mama, submetidas à quimioterapia/radioterapia, atendidas em um centro de câncer, na cidade de Fortaleza-CE, de julho a dezembro de 2012. Coletaram-se dados clínicos (subtipo, estadiamento clínico - EC, tratamento), socioeconômicos (escolaridade e renda mensal), antropométricos (peso, estatura, índice de massa corporal - IMC) e de consumo alimentar. Para avaliação do consumo, foi utilizado um questionário quantitativo de frequência alimentar (QQFA) validado para mulheres do Nordeste, e o consumo diário de cada alimento foi definido em gramas/ml, sendo agrupados, de acordo com sua composição nutricional, em 10 grupos, seguindo a pirâmide alimentar adaptada à população brasileira. Definiu-se o padrão alimentar por componentes alimentares a partir da análise fatorial explanatória dos dez grupos de alimentos, utilizando-se o SPSS, versão 20.0. Resultados: Idade média de 50,9 $\pm 10,2$ anos, com prevalência de carcinoma ductal $(n=83 ; 83 \%)$ em EC III ( $\mathrm{n}=60 ; 60 \%)$. A média do IMC indicou excesso de peso $\left(28,3 \pm 4,4 \mathrm{~kg} / \mathrm{m}^{2}\right)$ e a circunferência da cintura estava acima do recomendado $(98,6 \pm 10,9 \mathrm{~cm})$. Identificaram-se quatro componentes alimentares e $21 \%$ da variância da dieta foi explicada pelo componente alimentar 1 (Risk), com a matriz composta por carne vermelha e processada, óleos, gorduras e cereais. Conclusão: O padrão alimentar das pacientes foi caracterizado por uma dieta de risco, que pode contribuir positivamente para a recidiva da doença.

Descritores: Consumo de Alimentos; Câncer de Mama; Dieta; Avaliação Nutricional.

\section{ABSTRACT}

Objective: To identify a posteriori dietary pattern of women with breast cancer. Methods: Observational, cross-sectional and analytical study. It evaluated 100 women with breast cancer, undergoing chemotherapy/radiotherapy, treated at a cancer center in the city of Fortaleza, from July to December 2012. The study collected clinical data (subtype, clinical stage - CS, treatment), socioeconomic (educational level and monthly income) and anthropometric data (weight, height, body mass index - BMI), and food consumption. To evaluate the consumption, a quantitative food frequency questionnaire (FFQ), validated for women of the Brazilian Northeast, was applied, and the daily consumption of each food was set in grams $/ \mathrm{ml}$, being grouped according to their nutritional composition, into 10 groups, following the food pyramid adapted to the Brazilian population. The dietary pattern was defined by food components from the exploratory factor analysis of those ten food groups,

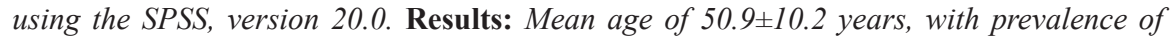
ductal carcinoma $(n=83 ; 83 \%)$ in CS III $(n=60 ; 60 \%)$. The mean BMI indicated overweight $\left(28.3 \pm 4.4 \mathrm{~kg} / \mathrm{m}^{2}\right)$ and waist circumference was above the recommended $(98.6 \pm 10.9 \mathrm{~cm})$. Four food components were identified, and $21 \%$ of the diet variance was explained by food component 1 (Risk), with the matrix composed of red and processed meat, oils, fats and cereals. Conclusion: The patients' dietary pattern was characterized by a risk diet, which can positively contribute to the disease recurrence.

Descriptors: Food Consumption; Breast Cancer; Diet; Nutrition Assessment
Artigo Original

1) Faculdade de Saúde Pública - FSP - São Paulo (SP) - Brasil

2) Universidade Estadual do Ceará - UECE - Fortaleza (CE) - Brasil

3) Universidade de Fortaleza - UNIFOR Fortaleza (CE) - Brasil

4) Centro Regional Integrado de Oncologia - CRIO - Fortaleza (CE) - Brasil

Recebido em: 24/03/2014 Revisado em: 20/05/2014 Aceito em: 05/07/2014 


\section{RESUMEN}

Objetivo: Identificar el patrón alimentario a posteriori de mujeres con cáncer de mama. Métodos: Estudio observacional, transversal y analítico. Fueron evaluadas 100 mujeres con cáncer de mama sometidas a quimioterapia/radioterapia asistidas en un centro de cáncer de Fortaleza-CE, de julio a diciembre de 2012. Se recogieron datos clínicos (subtipo, clasificación clínica $-C C$, tratamiento), socioeconómicos (escolaridad y renta mensual), antropométricos (peso, estatura, índice de masa corporal - IMC) $y$ de consumo alimentario. Para la evaluación del consumo, fue utilizado un cuestionario cuantitativo de frecuencia alimentaria (QQFA) validado para mujeres del Nordeste, y el consumo a diario de cada alimento fue definido en gramos $/ \mathrm{ml}$, siendo agrupados, según su composición nutricional, en 10 grupos, siguiendo la pirámide alimentaria adaptada a la población brasileña. Se definió el patrón alimentario por componentes alimentarios a partir del análisis factorial exploratorio de los diez grupos de alimentos, con la utilización del SPSS, versión 20.0. Resultados:

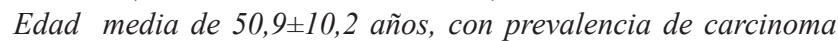
ductal ( $n=83 ; 83 \%)$ en CC III ( $n=60 ; 60 \%)$. La media del IMC indico exceso de peso $\left(28,3 \pm 4,4 \mathrm{~kg} / \mathrm{m}^{2}\right)$ y la circunferencia de la cintura estaba por encima del recomendado $(98,6 \pm 10,9 \mathrm{~cm})$. Se identificaron cuatro componentes alimentarios y el 21\% de la variancia de la dieta fue explicada por el componente alimentario 1 (Risk), con la matriz formada por carne roja y procesada, aceites, grasas y cereales. Conclusión: El patrón alimentario de las pacientes fue caracterizado por una dieta de riesgo que puede contribuir positivamente para la recidiva de la enfermedad.

Descriptores: Consumo de Alimentos; Neoplasias de la mama; Evaluación Nutricional.

\section{INTRODUÇÃO}

O câncer de mama é o segundo tipo de câncer mais frequente no mundo e o primeiro entre mulheres, sendo estimados para 2030 quase 27 milhões de novos casos, tornando-se, assim, um sério problema de saúde pública ${ }^{(1)}$. No Brasil, a estimativa para o ano de 2015 é de $576 \mathrm{mil}$ novos casos de neoplasia maligna da mama ${ }^{(1)}$.

A associação entre fatores dietéticos e câncer de mama é amplamente reconhecida na literatura. Porém, o papel da alimentação como facilitadora do desenvolvimento dessa neoplasia ainda não está claramente elucidado. Diversos estudos afirmam que a qualidade da alimentação, caracterizada por uma dieta "prudente", rica em frutas, legumes, cereais, azeite e produtos lácteos, parece exercer um possível efeito benéfico, contribuindo para a redução das taxas de incidência da doença ${ }^{(2,3)}$. Acredita-se que essa proteção pode ser atribuída ao consumo de nutrientes antioxidantes, como vitaminas (A, C e E) e os minerais zinco e selênio, considerados compostos bioativos dos alimentares capazes de reduzir significantemente os efeitos adversos produzidos pelas espécies reativas de oxigênio ${ }^{(3,4)}$. Apesar dessas evidências sobre os benefícios do consumo de vegetais na proteção contra o câncer de mama, uma relação inversa entre esse consumo regular e o desenvolvimento dessa neoplasia ainda é apresentada ${ }^{(5)}$.

Entendendo esses resultados controversos, é fundamental destacar que os indivíduos não consomem esses alimentos e nutrientes de forma isolada, e sim combinados em grandes refeições. E é essa combinação que promove a ação sinérgica de diversos compostos bioativos dos alimentos, o que parece interferir na transformação de uma célula saudável em uma célula doente ${ }^{(2,5,6)}$.

De tal modo, a caracterização qualitativa do padrão alimentar, e não apenas dos alimentos ou nutrientes isoladamente, pode fornecer subsídios importantes para a qualidade da dieta, sendo essa avaliação cada vez mais utilizada para analisar a associação entre as características qualitativas da dieta e o desenvolvimento das doenças crônicas ${ }^{(7)}$.

Assim, a análise de padrões alimentares tem sido uma abordagem recomendada para avaliar a relação entre a alimentação e o risco de doenças crônicas, como o câncer, que supera as limitações da abordagem de alimentos ou nutrientes específicos ${ }^{(7)}$. Nessa perspectiva, o objetivo deste trabalho foi identificar os padrões alimentares a posteriori de mulheres com câncer de mama.

\section{MÉTODOS}

Estudo observacional, transversal e qualitativo, realizado com mulheres com tumor da mama $(n=100)$, atendidas em um centro de oncologia na cidade de Fortaleza$\mathrm{CE}$, selecionadas por conveniência, de forma consecutiva e não probabilística, no período de julho a dezembro de 2012. Foram consideradas elegíveis as pacientes com mais de 19 anos, encaminhadas ao tratamento antineoplásico, sem orientação nutricional prévia. Vegetarianas, portadoras de neoplasias prévias ou associadas com câncer de mama e que já tinham concluído o tratamento há mais de dois anos não foram incluídas no estudo.

Os dados socioeconômicos e clínicos foram coletados por entrevista direta ou pesquisa ou prontuário, utilizando um questionário desenvolvido para esse fim, sendo obtidas informações sobre idade, escolaridade, renda mensal em salários mínimos (SM), localização do tumor, estadiamento clínico (EC) e tipo de tratamento antineoplásico.

Para a definição do estado nutricional, aferiu-se peso atual - PA (kg), estatura $(\mathrm{m})$ e circunferência de cintura - CC $(\mathrm{cm})$. O PA foi medido em uma balança plataforma da marca Plenna ${ }^{\circledR}$, com capacidade para $150 \mathrm{~kg}$, com 
as mulheres usando roupas leves e sem sapatos. Para a altura, usou-se um estadiômetro $2 \mathrm{~m}$, seguindo o plano de Frankfurt $^{(8)}$. A CC foi medida com fita métrica inelástica e flexível, sendo considerados elevados os valores $\geq 80 \mathrm{~cm}^{(9)}$.

Definiu-se o índice de massa corporal (IMC) pela fórmula peso/estatura ${ }^{(10)}$, utilizando-o como parâmetro para o diagnóstico nutricional, de acordo com a classificação da Organização Mundial da Saúde $(\mathrm{OMS})^{(11)}$ para mulheres adultas e para idosas $^{(12)}$.

Avaliou-se o padrão alimentar em duas etapas: a primeira constituiu-se da avaliação do consumo; a segunda contemplou a análise estatística.

O consumo alimentar foi avaliado por um entrevistador treinado, utilizando um questionário quantitativo de frequência alimentar (QQFA) validado para as mulheres do Nordeste $^{(13)}$ que contemplava 68 itens alimentares. A fim de minimizar o viés da memória, kits de medidas caseiras (pratos, xícaras, copos e talheres) característicos do uso popular da região, bem como registros fotográficos, foram usados para facilitar a quantificação das porções.

Definiu-se o consumo alimentar diário de cada paciente a partir do consumo habitual referido pelo QQFA, organizando-se os 68 itens alimentares em 10 grupos, de acordo com a pirâmide alimentar adaptada à população brasileira e também considerando a semelhança na composição nutricional.

Os grupos de alimentos definidos foram: Grupo 1 leite e derivados (leite, iogurte, coalhada, vitaminas, queijo); Grupo 2 - cereais (macarrão, lasanha, pizza, massas, arroz branco, batata doce, abóbora, batata ou mandioca, farinha, farinha de mandioca, cuscuz de milho, Neston, aveia, pão, biscoitos ou bolachas, milho, ervilha, vagem); Grupo 3 - carne vermelha e processada (carne, bife, carne seca, linguiça, presunto, ovo, miúdos de frango); Grupo 4 - carne branca (frango assado, peixe grelhado); Grupo 5 - frutas e sucos (laranja, tangerina, banana, mamão, maçã, melancia, melão, morango, abacaxi, pêssego, ameixa fresca, suco de laranja, suco de fruta natural); Grupo 6 - leguminosas (feijão); Grupo 7 - verduras e legumes (sopas, salada de maionese com legumes, tomate, alface, couve, espinafre, beterraba, cenoura, pepino, pimentão); Grupo 8 - óleos e gorduras (batatas fritas, mandioca frita, margarina no pão, manteiga no pão, azeite, maionese, peixe frito, frango frito); Grupo 9 - bebidas alcoólicas (cerveja, cachaça, uísque, vodka, vinho); Grupo 10 - doces e refrigerantes (açúcar adicionado ao leite, chocolates, doces, brigadeiros, bombons, bolos, tortas, sorvetes, refrigerantes, biscoito doce). Alguns alimentos foram excluídos porque não se encaixaram em nenhum grupo (café com açúcar, café sem açúcar, ketchup, mostarda, adoçante, água).
Os padrões alimentares foram obtidos através da análise fatorial exploratória dos dez grupos de alimentos ${ }^{(14)}$. A análise fatorial é um nome genérico dado à análise estatística multivariada aplicada à identificação de fatores em um conjunto de dados. Nesse método, todas as variáveis são consideradas simultaneamente, cada uma relacionada com a outra ${ }^{(14)}$.

Inicialmente, a fim de determinar a adequação da utilização da análise fatorial, a uniformidade da amostra foi testada para examinar a distribuição das variáveis, através do teste de Kaiser-Meyer-Olkin (KMO). A presença de correlação entre as variáveis seguiu o teste de esfericidade de Bartlett, para o qual foram considerados aceitáveis os valores de KMO acima de 0,50 e valores de $\mathrm{p}<0,05^{(15)}$.

A análise do componente principal foi utilizada para extrair os fatores. Esse método examina a distribuição espacial dos objetos, a fim de identificar os grupos e as relações entre eles. O primeiro fator ou componente extraído explica a maior variância possível no conjunto de dados. $\mathrm{O}$ segundo componente, independente do primeiro, explica a maior parte da variância restante, e assim por diante, sem qualquer correlação com o outro.

A escolha do número de fatores foi primeiro baseado no critério de Kaiser, chamado de eigenvalues acima de 1,0. É o critério mais utilizado na análise fatorial, e a base teórica por trás dele é que cada fator retido deve explicar a maior variância no conjunto de dados original. Foram identificados quatro fatores, de acordo com o critério de eigenvalue $>1,0$ aplicado. Os padrões foram nomeados considerando os grupos de alimentos que tiveram valores mais elevados (positivos ou negativos), ao longo de 0,5. Maiores valores positivos indicam uma forte associação entre os grupos e padrões. A análise estatística foi realizada utilizando o programa SPSS, versão 20.0.

Este estudo obedeceu à Resolução 466/12 do Conselho Nacional de Saúde, sendo aprovado pelo Comitê de Ética em Pesquisa da Universidade de Fortaleza (parecer no 204/10), e teve a coleta de informações iniciada após as pacientes darem seu consentimento informado por escrito.

\section{RESULTADOS}

A média de idade foi de 50,9 $\pm 10,2$ anos (27-78 anos), a maioria das mulheres tinha ensino fundamental $(n=54 ; 54 \%)$ e renda mensal inferior a um salário mínimo $(n=59 ; 59,5 \%)$. A maior prevalência foi de carcinoma ductal $(n=83 ; 83 \%)$ e estadiamento clínico (EC) III ( $n=60 ; 60 \%)$. A quimioterapia associada à radioterapia foi o tratamento mais frequente, usado em 45 (45\%) pacientes (Tabela I).

O IMC médio foi de $28,3 \pm 4,4 \mathrm{~kg} / \mathrm{m}^{2}\left(20-40 \mathrm{~kg} / \mathrm{m}^{2}\right)$ e a média da circunferência da cintura foi de $98,6 \mathrm{~cm}$ (62- 
Tabela I - Descrição das pacientes de acordo com o perfil socioeconômico e clínico. Fortaleza-CE, 2012.

\begin{tabular}{|c|c|c|}
\hline Variáveis & $\mathbf{n}$ & $\%$ \\
\hline \multicolumn{3}{|l|}{ Escolaridade } \\
\hline Alfabetizado & 6 & $6 \%$ \\
\hline Ensino fundamental incompleto & 54 & $54 \%$ \\
\hline Ensino fundamental completo & 8 & $8 \%$ \\
\hline Ensino médio incompleto & 4 & $4 \%$ \\
\hline Concluído o ensino médio & 14 & $14 \%$ \\
\hline Nível superior completo & 9 & $9 \%$ \\
\hline Em falta & 5 & $5 \%$ \\
\hline \multicolumn{3}{|l|}{ Renda mensal } \\
\hline$<1 \mathrm{sm}^{*}$ & 59 & $59 \%$ \\
\hline $1-3 \mathrm{sm}^{*}$ & 28 & $28 \%$ \\
\hline$>3 \mathrm{sm}^{*}$ & 6 & $6 \%$ \\
\hline Sem renda & 7 & $7 \%$ \\
\hline \multicolumn{3}{|l|}{ Subtipo } \\
\hline Ductal & 83 & $83 \%$ \\
\hline Lobular & 3 & $3 \%$ \\
\hline Outros & 1 & $1 \%$ \\
\hline Não informado** & 13 & $13 \%$ \\
\hline \multicolumn{3}{|l|}{ Estágio clínico } \\
\hline I & 8 & $8 \%$ \\
\hline II & 16 & $16 \%$ \\
\hline III & 60 & $60 \%$ \\
\hline IV & 3 & $3 \%$ \\
\hline Não informado** & 13 & $13 \%$ \\
\hline \multicolumn{3}{|l|}{ Tratamento } \\
\hline Quimioterapia & 24 & $24 \%$ \\
\hline Radioterapia & 26 & $26 \%$ \\
\hline Quimioterapia + radioterapia & 45 & $45 \%$ \\
\hline Não informado** & 5 & $5 \%$ \\
\hline
\end{tabular}

*Salário mínimo: \$311,74; ** Informação não encontrada no prontuário do paciente.

$128 \mathrm{~cm})$. De acordo com o IMC, o diagnóstico nutricional da maioria das pacientes indicou excesso de peso (sobrepeso e obesidade) ( $\mathrm{n}=76 ; 76 \%)$ (Figura 1), e $89(89 \%)$ mulheres apresentaram circunferência da cintura acima do recomendado.

Com relação à avaliação do padrão alimentar a posteriori, a partir da análise de componentes principais, quatro fatores independentes foram extraídos, com critério eigenvalue $>1,0$ (Tabela II). Esses fatores foram nomeados componentes alimentares e numerados em 1, 2, 3 e 4 . O primeiro componente alimentar teve sua matriz composta pelos grupos "carnes vermelhas e processadas" $(0,672)$, "óleos e gorduras" $(0,707)$ e "cereais" $(0,683)$, sendo nomeado como "Risco". A matriz do segundo componente foi explicada pelos grupos alimentares "leite e derivados" $(0,653)$, "frutas e sucos" $(0,546)$ e "verduras e legumes" $(0,780)$, sendo chamado de "Protetor". O terceiro componente foi representado por "carnes brancas" $(0,580)$ e "leguminosas" $(0,850)$, sendo nomeado de "Tradicional". E o quarto componente, caracterizado pela predominância de "álcool" $(0,722)$, "doces e refrigerantes" $(0,636)$, foi chamado de "Não saudável". Esses fatores foram responsáveis por $60,1 \%$ da variância total da ingestão de alimentos dessas pacientes (Tabela II).

O componente "Risco" foi responsável por $21 \%$ da variância total da dieta, enquanto o componente "Protetor" explicou $15,8 \%$ do padrão alimentar (Tabela II). 


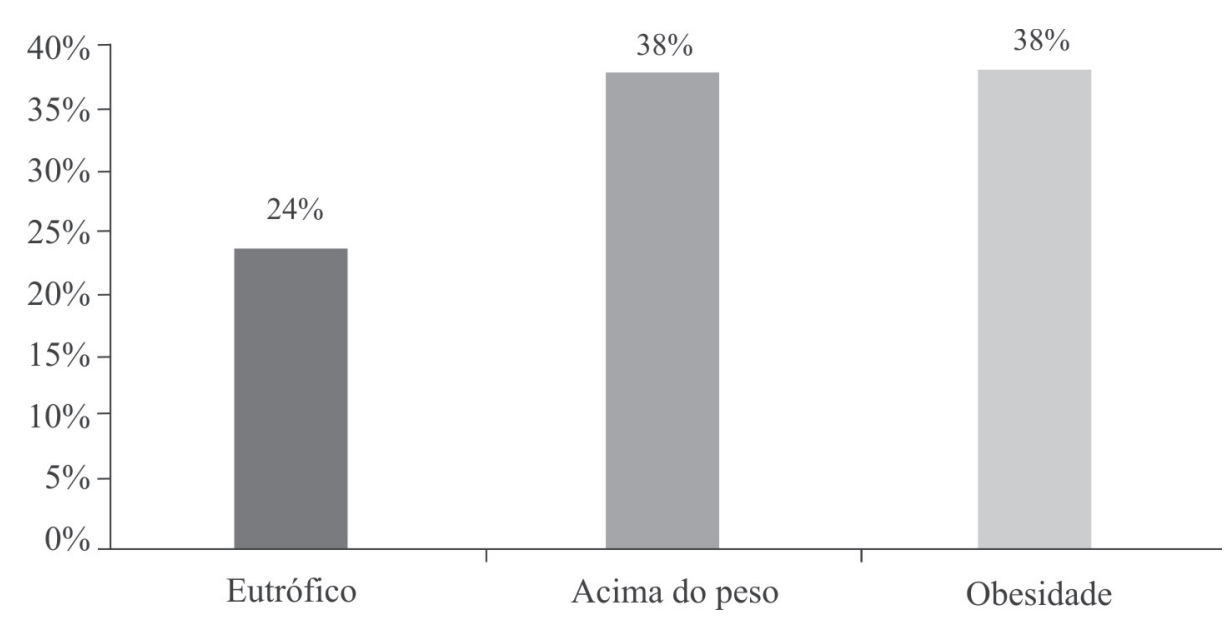

Figura 1 - Descrição do percentual das pacientes de acordo com o estado nutricional definido pelo Índice de Massa Corporal (IMC). Fortaleza-CE, 2012.

Tabela II - Descrição do padrão alimentar identificado pelo Questionário Quantitativo de Frequência Alimentar (QQFA) usando análise fatorial sobre a população total. Fortaleza-CE, 2012.

\begin{tabular}{lcccc}
\hline \multirow{2}{*}{ Grupo alimentar } & \multicolumn{3}{c}{ Padrão alimentar } \\
\cline { 2 - 5 } & $\begin{array}{c}\text { Componente 1: } \\
\text { Risco }\end{array}$ & $\begin{array}{c}\text { Componente 2: } \\
\text { Protetor }\end{array}$ & $\begin{array}{c}\text { Componente 3: } \\
\text { Tradicional }\end{array}$ & $\begin{array}{c}\text { Componente 4: } \\
\text { Não saudável }\end{array}$ \\
\hline Leite e produtos lácteos & $-0,311$ & 0,653 & 0,164 & $-0,043$ \\
Cereais & 0,683 & 0,208 & 0,185 & 0,031 \\
Carne vermelha e processada & 0,672 & 0,193 & $-0,260$ & 0,224 \\
Carne branca & 0,348 & 0,373 & 0,580 & $-0,104$ \\
Frutas e sucos & 0,165 & 0,546 & 0,085 & 0,327 \\
Leguminosas & $-0,013$ & $-0,008$ & 0,850 & 0,122 \\
Verduras e legumes & 0,201 & 0,780 & $-0,069$ & $-0,171$ \\
Óleos e gorduras & 0,707 & $-0,279$ & 0,138 & $-0,073$ \\
Bebidas alcoólicas & $-0,194$ & $-0,052$ & 0,332 & 0,722 \\
Doces e refrigerantes & 0,335 & $-0,010$ & $-0,221$ & 0,636 \\
\hline \% variância explicada & 21,0 & 15,8 & 12,9 & 10,3 \\
\hline
\end{tabular}

\section{DISCUSSÃO}

Trata-se de um estudo pioneiro em avaliar o padrão alimentar a posteriori de mulheres com câncer de mama, no Nordeste. E os resultados mostraram que as pacientes avaliadas apresentaram padrão alimentar de risco, composto pelo consumo de carne vermelha, embutidos, óleos, gorduras, cereais, além da elevada prevalência de excesso de peso e gordura abdominal.

$\mathrm{O}$ valor elevado do IMC tem sido associado ao risco aumentado do câncer de mama em mulheres na pósmenopausa ${ }^{(16)}$, e evidências substanciais insinuam que mulheres obesas têm menor sobrevida após o diagnóstico de câncer de mama do que as mulheres com peso adequado ${ }^{(17)}$. Além disso, a medida da circunferência da cintura acima do recomendado, associada à gordura localizada na região abdominal, parece atuar como importante fator de risco para o desenvolvimento de alguns tipos de câncer ${ }^{(18)}$. Esses achados sugerem que as pacientes do presente estudo, entre as quais $76,7 \%$ estavam com excesso de peso e $89 \%$ com $\mathrm{CC}$ acima do recomendado, apresentam variáveis alteradas que podem contribuir para possível recorrência do câncer de mama.

Apesar das evidências existentes, o mecanismo causal, através do qual o excesso de peso e tecido adiposo afeta o prognóstico do câncer, permanece por ser elucidado, mas tem sido sugerido estar relacionado ao papel endócrino complexo e metabolicamente ativo exercido pelo tecido adiposo, que se mostra integralmente envolvido na coordenação de vários processos biológicos ${ }^{(19)}$. 
Quando esse excesso se caracteriza por adiposidade visceral, medida pela CC, cria-se um ambiente mais favorável para o desenvolvimento do tumor, pois o tecido adiposo visceral está envolvido em um quadro de inflamação crônica de baixa intensidade, que mobiliza macrófagos, promove elevação dos níveis de fator de necrose tumoral (TNF) e interleucinas (IL), leva à hiperinsulinemia e aumento dos níveis de fator de crescimento semelhante à insulina (IGF-1), findando com estado pró-tumorigênico de inflamação, angiogênese e resistência à insulina ${ }^{(20,21,22)}$.

Em concordância com as alterações metabólicas carcinogênicas, na presença do excesso de peso, há também aumento dos níveis circulantes de estrogênio, possivelmente através do aumento da massa gorda, e o aumento da expressão da aromatase no tecido adiposo subcutâneo, os quais, associados aos níveis plasmáticos reduzidos de globulina ligante de hormônio sexual (SHGB), permitem que o estrógeno permaneça livre e atue sobre a célula do epitélio mamário ${ }^{(20,21)}$.

Uma vez que o excesso de peso é visto como um importante fator prognóstico negativo para a sobrevida de mulheres com câncer de mama e que também há relação com a progressão ou recidiva da doença, é importante manter o peso corporal dentro dos limites normais e evitar o ganho de peso e aumento da circunferência da cintura ao longo da idade adulta. Para isso, é imprescindível a adoção de um estilo de vida saudável, manter-se fisicamente ativo e adotar bons hábitos alimentares ${ }^{(22,23)}$.

Nesse ínterim, tem sido amplamente discutida a importância de se analisar a relação entre dieta e câncer usando o padrão alimentar, e não somente considerando os nutrientes ou alimentos de forma específica. Acreditase que essa análise pode ser mais confiável, uma vez que os alimentos não são consumidos isoladamente, mas em refeições que consistem em combinações desses alimentos $^{(24) .}$ Além disso, estudos têm demonstrado que a análise de padrões alimentares é melhor preditora do risco de doença, pois reduz as variáveis em grupos significativos e mais fáceis de analisar ${ }^{25)}$.

No presente estudo, verificou-se que o padrão alimentar das pacientes segue o consumo de uma dieta de risco, na qual grupos alimentares compostos de carnes vermelhas e processadas, óleos, gorduras e cereais constituem a maior percentagem da alimentação. Assim, o componente de risco reúne alimentos que podem atuar como fatores de risco para a recidiva do câncer de mama, especialmente se a dieta está associada ao diagnóstico nutricional de obesidade. Vêse a presença de cereais entre os grupos alimentares das pacientes avaliadas. Porém, considerando as características dos alimentos da região Nordeste, crê-se que os cereais consumidos devem ter sido do tipo refinado, já que o consumo de alimentos integrais, embora estimulado por programas do governo, não é rotineiramente adotado como hábito alimentar nessa população.

O papel da dieta na etiologia do câncer de mama tem sido sugerido, em parte, devido à grande variação nas taxas internacionais de câncer. Ele pode ser atribuído às propriedades antioxidantes dos nutrientes selecionados, sua influência sobre a resposta inflamatória e imunitária celular na progressão através do ciclo celular e na reparação do DNA, às mutações do DNA, à desintoxicação metabólica, à estimulação de fatores de crescimento e à potencial influência antiestrogênica de alguns nutrientes ${ }^{(26)}$.

Uma dieta com elevado consumo de frutas, legumes e cereais integrais, juntamente com nutrientes antioxidantes, fibras e fitoquímicos, tem ação preventiva ${ }^{(22)}$. Por outro lado, a ingestão excessiva de alimentos ricos em gordura saturada, poli-insaturada e trans, tais como

carne vermelha, frituras, leite e derivados, bacon, presunto, salsichas e mortadela, é considerada de risco para o desenvolvimento da doença $a^{(7,27,28)}$. De modo antagônico, um estudo realizado com mulheres com câncer de mama concluiu que uma maior adesão aos hábitos alimentares saudáveis, com dieta rica em frutas, vegetais, grãos integrais, aves, peixes e produtos lácteos com baixo teor de gordura, foi associada à diminuição da mortalidade geral ${ }^{(28)}$.

Por conseguinte, adotar uma dieta que consiste principalmente em frutas, legumes, peixe e azeite de oliva/ óleo de girassol, e, além disso, evitar alimentos como carne vermelha, alimentos processados e enlatados, ricos em gordura, cereais refinados e bebidas alcoólicas pode contribuir para uma redução substancial do risco de câncer de mama ${ }^{(29)}$.

O consumo de carne vermelha tem sido fortemente associado ao risco aumentado de câncer de mama, especialmente em mulheres na pré-menopausa ${ }^{(29)}$. Da mesma forma, mulheres cujo padrão alimentar é caracterizado pelo elevado consumo de cereais refinados, carnes vermelhas e picles têm maior risco de desenvolver a doença ${ }^{(30)}$. A relação entre o consumo de carnes vermelhas e carnes processadas com o câncer de mama pode ser explicada pela alta quantidade de gorduras presentes nesses alimentos, podendo estimular a peroxidação lipídica e promover o estresse oxidativo nessas pacientes ${ }^{(31)}$. Igualmente, a gordura saturada da carne vermelha parece estimular a produção de estrógenos endógenos, que induzem a proliferação de células epiteliais mamárias. Aliado a isso, o colesterol está associado ao aumento da densidade da mama, o que também é um fator de risco para o desenvolvimento da doença. A carne vermelha tem potentes agentes cancerígenos, como aminas heterocíclicas, hidrocarbonetos aromáticos policíclicos e outros compostos nitrogenados, que também podem aumentar o risco da neoplasia ${ }^{(32)}$. 
Reforçando essa ideia, um estudo realizado com mulheres que tiveram o diagnóstico precoce de câncer de mama descobriu que o padrão alimentar - o qual consiste em consumo elevado de grãos refinados, doces, carnes vermelhas e processadas, laticínios ricos em gordura, snacks e manteiga - foi relacionado a um maior risco de morte por todas as causas e mortalidade por câncer de mama ${ }^{(17)}$.

O segundo componente alimentar ("Protetor") encontrado neste estudo foi caracterizado por grupos de laticínios, frutas, sucos e legumes. Esse é o componente que tem a segunda representação no padrão alimentar dessas mulheres, por isso uma menor percentagem. Padrão alimentar rico em frutas e saladas pode ser protetor contra o câncer de mama invasivo, e esse efeito benéfico deve ser maior para os tumores receptor de estrógeno (ER) e receptor de progesterona negativo (PR) ${ }^{(33)}$. No entanto, tal padrão protetor não foi o encontrado no presente estudo, uma vez que o maior percentual de variação é explicado pelo componente de risco.

Atualmente, existem evidências consistentes de que um padrão alimentar saudável (rico em alimentos vegetais e pobre em carnes vermelhas e processadas) está associado a um risco reduzido de câncer de mama ${ }^{(34)}$. Reforçando essa hipótese, um padrão alimentar caracterizado pelo consumo de legumes, frutas, azeite de oliva, peixe, soja e baixa ingestão de gordura está associado negativamente ao câncer de $\operatorname{mama}^{(2)}$.

Entende-se que a avaliação do consumo alimentar é sempre um importante fator limitante nos estudos que buscam avaliar a relação dieta-doença, afinal, as escolhas alimentares não são definidas apenas pelo conhecimento que o indivíduo tem sobre o que é saudável, pois dependem de aspectos sociais, econômicos e afetivos. Assim, uma das limitações do presente estudo é o fato de ter sido realizado apenas com pacientes integrantes da classe social de baixa renda, o que limita muitas escolhas alimentares. Portanto, recomenda-se a ampliação do estudo também para as populações de média e alta renda, a fim de inserir na investigação desse padrão alimentar outros níveis sociais e definir o padrão alimentar dessas mulheres diagnosticadas com câncer de mama que compõem a população avaliada.

Conhecer esse padrão alimentar das mulheres do Nordeste de modo mais aprofundado certamente contribuirá para promover o consumo de uma dieta protetora, considerando os alimentos regionais e as condições socioeconômicas de onde essas pacientes estão inseridas.

\section{CONCLUSÃO}

O padrão alimentar das pacientes avaliadas foi representado principalmente pelo consumo de carne vermelha e processada, óleos, gorduras e cereais, caracterizando uma dieta de risco, que pode contribuir positivamente para a recidiva da doença.

\section{AGRADECIMENTOS}

Ao CRIO pelo apoio e também às pacientes voluntárias.

\section{REFERÊNCIAS}

1. Instituto Nacional do Câncer José Alencar Gomes da Silva - INCA. Estimativa 2014: Incidência de Câncer no Brasil. Rio de Janeiro: INCA; 2014.

2. Collins TA, Rosenberg L, Makambi K, Palmer JR, Adams CL. Dietary patterns and breast cancer risk in women participating in the Black Women's Health Study. Am J Clin Nutri. 2009;90(3):621-8.

3. Rock CL, Doyle C, Demark-Wahnefried W, Meyerhardt J, Courneya KS, Schwartz AL et al. Nutrition and physical activity guidelines for cancer survivors. CA Cancer J Clin. 2012; 62(4):243-74

4. Sacheck JM, Blumberg JB. Vitamins and oxidative stress. Boston: USDA Human Nutrition Research Center on Aging; 2013

5. Kolling, FL, Santos JS. A influência dos fatores de risco nutricionais no desenvolvimento de câncer de mama em pacientes ambulatoriais do interior do Rio Grande do Sul, Brasil. Sci Med. 2009;19(3):115-21.

6. Brennan SF, Cantwell MM, Cardwell CR, Velentzis LS, Woodside JV. Dietary patterns and breast cancer risk: a systematic review and meta-analysis. Am J Clin Nutri. 2010;91(5):1294-302.

7 Azevedo ECC, Diniz AS, Monteiro JS, Cabral PC. Padrão alimentar de risco para as doenças crônicas não transmissíveis e sua associação com a gordura corporal: uma revisão sistemática. Ciênc Saúde Coletiva. 2014;19(5):1447-58.

8 Dias MCG, Horie LM, Waitzberg DL. Exame físico e antropometria. In: Waitzberg DL. Nutrição oral, enteral e parenteral na prática clínica. $4^{\mathrm{a}}$ ed. São Paulo: Atheneu; 2002. p. 383-419.

9 World Health Organization - WHO. Report of a Joint FAO/WHO Consultation. Preparation and use of foodbased dietary guidelines. Geneva; 1998.

10. Cibeira GH, Guaragna RM. Lipídio: fator de risco e prevenção do câncer de mama. Rev Nutr. 2006;19(1):65-75. 
11. World Health Organization - WHO. Obesity: preventing and managing the global epidemic. Report of a WHO Consultation. Geneva; 2000. (Technical Report Series 894).

12. Lipschitz DA. Screening for nutritional status in the elderly. Prim Care. 1994;21(1): 55-67.

13. Lima FEL, Slater B, Latorre MRDO, Fisberg RM. Validade de um questionário quantitativo de freqüência alimentar desenvolvido para população feminina no nordeste do Brasil. Rev Bras Epidemiol. 2007;10(4):483-90.

14. Marchioni DML, Latorre MRDO, Eluf-Neto J, Wünsch-Filho V, Fisberg RM. Identification of dietary patterns using factor analysis in an epidemiological study in São Paulo. Sao Paulo Med J. 2005;123(3):12427.

15. Hair JJ, Anderson RE, Tatham RL. Análise multivariada de dados. $5^{\mathrm{a}}$ ed. Porto Alegre: Bookman; 2005.

16. Cecchini RS, Costantino JP, Cauley JA, Cronin WM, Wickerham DL, Land SR, et al. Body Mass Index and the Risk for Developing Invasive Breast Cancer among High-Risk Women in NSABP P-1 and STAR Breast Cancer Prevention Trials. Cancer Prev Res. 2012;5(4):583-92.

17. Kwan ML, Chen WY, Kroenke CH, Weltzien EK, Beasley JM, Nechuta SJ, et al. Pre-diagnosis body mass index and survival after breast cancer in the after breast cancer pooling project. Breast Cancer Res Treat. 2012;132(2):729-39.

18. Menezes TN, Rocha FL, Belém PLO, Pedraza DF. Obesidade abdominal: revisão crítica das técnicas de aferição e dos pontos de corte de indicadores antropométricos adotados no Brasil. Ciênc Saúde Coletiva. 2014;19(6):1-14.

19. Ewertz M, Gray KP, Regan MM, Ejlertsen B, Price $\mathrm{KN}$, Thürlimann B, et al. Obesity and Risk of recurrence or death after adjuvant endocrine therapy with letrozole or tamoxifen in the Breast International Group 1-98 Trial. J Clin Oncol. 2012;30(32):3967-75.

20. Garrisi VM, Tufaro A, Trerotoli P, Bongarzone I, Quaranta M, Ventrella V, et al. Body mass index and serum proteomic profile in breast cancer and healthy women: a prospective study. PLoS One. 2012; $7(11): 1-5$.

21. Chen D, Zhao H, Coon VJS, Ono M, Pearson EK, Bulun SE. Weight gain increases human aromatase expression in mammary gland. Mol Cell Endocrinol. 2012; 355(1):114-20.

22. Rubin BA, Stein AT, Zelmanowicz AM, Rosa DD. Perfil antropométrico e conhecimento nutricional de mulheres sobreviventes de câncer de mama do Sul do Brasil. Rev Bras Cancerol. 2010;56(3):303-9.

23. Fung TT, Schulze MB, Hu FB, Hankinson SE, Holmes MD. A dietary pattern derived to correlate with estrogens and risk of postmenopausal breast cancer. Breast Cancer Res Treat. 2012;132(3):1157-62.

24. Isabelle R. Diet and breast cancer. Salud Pública Mex. 2011;53(5):430-9.

25. Abdull Razis AF, Noor NM. Cruciferous vegetables: dietary phytochemicals for cancer prevention. Asian Pac J Cancer Prev. 2013;14(3):1565-70.

26. Demetriou CA, Hadjisavvas A, Loizidou MA, Loucaides G, Neophytou I, Sieri S, et al. The mediterranean dietary pattern and breast cancer risk in Greek-Cypriot women: a case-control study. BMC Cancer. 2012;12(113):1-12.

27. Kwan ML, Weltzien E, Kushi LH, Castillo A, Slattery ML, Caan BJ. Dietary patterns and breast cancer recurrence and survival among women with earlystage breast cancer. J Clin Oncol. 2009;27(6):919-26.

28. Cottet V, Touvier M, Fournier A, Touillaud MS, Lafay L, Clavel-Chapelon F, et al. Postmenopausal breast cancer risk and dietary patterns in the E3NEPIC prospective cohort study. Am J Epidemiol. 2009; 170(10):1257-67.

29. Taylor VH, Misra M, Mukherjee SD. Is red meat intake a risk factor for breast cancer among premenopausal women? Breast Cancer Res Treat. 2009;117(1):1-8.

30. Zhang CX, Ho SC, Fu JH, Cheng SZ, Chen YM, Lin FY. Dietary patterns and breast cancer risk among Chinese women. Cancer Causes Control. 2011;22(1):115-24.

31. Rockenbach G, Di Pietro PF, Ambrosi C, Boaventura BCB, Vieira FGK, Crippa CG, et al. Dietary intake and oxidative stress in breast cancer: before and after treatments. Nutr Hosp. 2011;26(4):737-44.

32. Marques AC, Valente TB, Rosa CS. Formação de toxinas durante o processamento de alimentos e as possíveis consequências para o organismo humano. Rev Nutr. 2009; 2(22):283-93.

33. Baglietto L, Krishnan K, Severi G, Hodge A,Brinkman M, English DR et al. Dietary patterns and risk of breast cancer. Br J Cancer. 2011;104(3):524-31. 
34. Brennan SF, Cantwell MM, Cardwell CR, Velentzis LS, Woodside JV. Dietary patterns and breast cancer risk: a systematic review and metaanalysis. Am J Clin Nutr. 2010;91(5):1294-302.

\section{Endereço para correspondência:}

Karin Sedó Sarkis

Rua Haddock Lobo, 1336, 41B

Bairro: Cerqueira César

CEP: 01414-002 - São Paulo - SP - Brasil

E-mail: karinsarkis@gmail.com 